\title{
An Economic Analysis of the Impact of Homecare Drug Administration for Biologic Interventions Available for Plaque Psoriasis in the UK
}

\author{
William Green (D) · Richard Stork · Aina Pi Blanque · Ayeda Nadeem
}

Received: June 5, 2021 / Accepted: July 10, 2021 / Published online: July 23, 2021

(c) The Author(s) 2021

\begin{abstract}
Introduction: In the UK, biologic interventions for plaque psoriasis can either be administered in a hospital setting or following delivery to a patient's home. To date, limited research has been undertaken on how the administration route affects the overall treatment costs and the implications for this on UK clinical practice. The objective was to explore the cost implications of different administration routes for plaque psoriasis biologic interventions in the UK.

Methods: A simple economic model was developed to estimate and compare the total cost of drug administration over 2 years for all biologic interventions that have been approved by the National Institute of Health and Care Excellence for use in patients with moderate-tosevere plaque psoriasis. Administration costs were estimated for two different scenarios: administration in a hospital setting or following home delivery [paid for by the National Health Service (NHS)].
\end{abstract}

W. Green $(\varangle) \cdot$ A. Nadeem

York Health Economics Consortium, York, UK

e-mail: william.green@york.ac.uk

R. Stork

Almirall, Uxbridge, UK

A. P. Blanque

Almirall S.A, Barcelona, Spain
Results: Costs of home delivery and administration in hospital over a 2-year time horizon varied substantially based on the choice of intervention. For home delivery, the lowest cost of $£ 693$ occurred with risankizumab, tildrakizumab and ustekinumab, while the highest cost of $£ 3445$ occurred with adalimumab, brodalumab, certolizumab and etanercept. For the scenario in which the interventions were administered in a hospital setting the costs ranged from $£ 4224$ for ustekinumab to $£ 7463$ for brodalumab.

Conclusion: These results indicate that drug administration costs are meaningful and should be given greater consideration in the selection process of treatments for plaque psoriasis. Additionally, the NHS could save money by paying for drugs to be delivered to a patient's home, rather than administering them in a hospital setting.

Keywords: Plaque psoriasis; Biologic interventions; Drug administration; Home delivery 


\section{Key Summary Points}

There is limited research on how administration of biologic interventions for plaque psoriasis affects the overall treatment costs.

This analysis explores the cost implications of different administration routes for plaque psoriasis biologic interventions in the UK.

Administration costs of biologic interventions for plaque psoriasis are meaningful and should be given greater consideration in the treatment selection process.

\section{INTRODUCTION}

Psoriasis is a chronic inflammatory skin disease which follows an unpredictable course with flare-ups and remissions [1, 2]. An estimated $2.5 \%$ of the population in England and Wales have psoriasis, of whom about $20 \%$ have a moderate-to-severe form of the disease [2, 3]. Additionally, approximately $90 \%$ of people with psoriasis have red, scaly plaques on the skin, which typically affect the knees, elbows, trunk and scalp [2]. Symptoms of the plaques can include itchiness, bleeding and scaling, which can cause severe physical and emotional discomfort [4, 5]. Associated morbidity with plaque psoriasis can be substantial, with patients suffering from a decreased quality of life $[1,4]$. Reduced levels of employment and income due to the condition can negatively affect the emotional, psychological and social wellbeing of patients [4]. Furthermore, patients with moderate-to-severe plaque psoriasis have an increased risk of psoriatic arthritis, cardiovascular disease, psychological disorders (such as anxiety and depression) and some forms of skin cancer $[4,6]$.

Although there is no cure for psoriasis, there are many treatments that can help with long- term management of the condition. These can include topical treatments such as emollients and occlusive dressings, phototherapy and systemic therapies such as biologics [2]. Treatment of plaque psoriasis is based on various factors including the severity of the plaques and patient response to prior treatment. In some cases, topical treatments are not well tolerated or have contraindications [4]. Studies have shown that a combination of phototherapy and systemic therapy is necessary in the treatment of patients with moderate-to-severe psoriasis $[4,7]$.

In recent years, several systemic therapies in the form of biologics have been developed and approved for the treatment of psoriasis. Generally, the aim of these therapies are to target pro-inflammatory cytokines, which, in turn, reduces the symptoms of the condition [8]. The following biologics are currently approved in the UK by the National Institute of Health and Care Excellence (NICE) for the treatment of plaque psoriasis when other systemic therapies have failed or are not well tolerated: etanercept, adalimumab, ustekinumab, secukinumab, ixekizumab, brodalumab guselkumab, tildrakizumab, risankizumab and certolizumab [2].

Due to the nature of biologic interventions, consideration must be given in terms of how they are administered to patients (e.g. storage and delivery method). Current administration options in the UK are administration in hospitals or via home delivery systems. This second option can either be funded by the National Health Service (NHS) or by manufacturers distributing the intervention [9]. Recently, the NHS has been investigating the possibility of taking over the home delivery of biologics such that this is funded by the NHS rather than by the drug manufacturers. A rapid guideline produced by NICE highlights options for delivering treatment during the coronavirus disease 2019 (COVID-19) pandemic [10]. This included the recommendation that face-to-face contact be avoided by using different methods to deliver treatment, such as postal services, NHS Volunteer Responders or by using drive-through pickup points for medications. 
A rapid review of previous NICE submissions for biological therapies within the area of plaque psoriasis [11-18] indicates that the cost of drug delivery has been overlooked (i.e. it was implicitly assumed that the cost of drug delivery was equal across all potential interventions). However, unless delivery is facilitated by the distributing company, there will be cost associated with drug delivery to the healthcare system, and this may vary by biologic intervention owing to differences in dosing frequency. Thus, the aim of the current study is to explore the potential cost implications of different options for delivering biological interventions to plaque psoriasis patients in the UK. The focus is on delivering interventions through home delivery systems, but the study also considers administration in hospitals.

\section{METHODS}

Previously, a large number of economic models have been developed to explore to cost-effectiveness of plaque psoriasis treatments in the $\mathrm{UK}$, including those developed to inform submissions to NICE [11-18]. In general, a consistent structure has been developed for these submissions, namely, a Markov model that aimed to quantify the cost and health implications of interventions by tracking movement between health states defined by psoriasis area and severity index (PASI) score. Additionally, these models generally explored the impact of incorporating interventions as part of treatment sequences. An Excel-based economic model from the perspective of the UK NHS was developed for the purpose of this study. However, given the aim of the study was to explore the cost implications of drug delivery/administration options only, a simple cost analysis model was developed as a more complex structure was judged to be unnecessary. Therefore, no consideration was given to health outcomes or the impact of sequences, and a Markov structure was not required. Additionally, the wider cost implications, such as the impact of each intervention in the context of non-delivery/administration costs, were not considered. In particular, the analysis did not calculate the impact of the cost of the interventions themselves. The intervention costs were excluded from the analysis because confidential patient access scheme (PAS) discounts have been agreed for a large number of the interventions included in the analysis (e.g. secukinumab, brodalumab, ixekizumab). As these discounts are confidential, it is not possible to know the true price of these interventions in the UK currently.

The total drug delivery costs were estimated over a 2-year time horizon for one hypothetical patient receiving each included intervention. The model started at the point at which the intervention is first administered, and therefore, the induction period for each drug was captured. To simplify the analysis, it was assumed that the patient remained on the intervention for the full 2-year period. In reality, a proportion of patients will discontinue from biological therapies each year, in particular if the intervention does not achieve an adequate response after the initial induction period. However, if a patient does achieve an initial response, these biologic interventions are generally well tolerated, and therefore, patients are likely to remain on the treatment for an extended period of time. Therefore, it is plausible for someone to receive a biologic intervention for a 2-year period.

Two separate scenarios were considered in the analysis: administration of the intervention in hospitals and home delivery paid by the NHS. Further details are provided below.

A key input within the model was the frequency of administration for each intervention, as this determines how often drug delivery is required (i.e. a greater frequency of administration was expected to lead to higher delivery costs). The frequency of administration for each intervention was sourced from the British National Formulary (BNF) [19].

In terms of home delivery, it was necessary to make a small number of assumptions in order to estimate the total cost of this option. First, it was assumed that a nurse would travel to a patient's home to administer the first dose of the intervention, and this would, on average, require a total of $1 \mathrm{~h}$ of nurse time (including travel). The total cost of such a nurse visit was 
estimated to be $£ 140 .{ }^{1}$ Second, it was assumed that all patients would self-administer the drug after the first dose and, therefore, subsequent nurse time would not be required. The unit cost of delivering the drug without a nurse was estimated to be $£ 65$, to account for the transfer of the intervention to the patient's home (see Footnote 1). Therefore, the total cost of the drug delivery was estimated to be $£ 140$ for the first dose and $£ 65$ for each subsequent dose (i.e. when the nurse is no longer required). The total cost of home delivery for each intervention was, thus, calculated by multiplying the dosing frequency over 2 years with the delivery costs just outlined.

For the administration in hospital delivery method, it was assumed a patient would travel to hospital and be given the intervention by a nurse during a 20-min outpatient appointment. The unit cost of this activity was sourced from the Personal Social Services Research Unit (PSSRU) and equated to a value of $£ 47$ for $1 \mathrm{~h}$ with a band 6 nurse [20]. This unit cost was multiplied by the time required for administration, giving a total cost of $£ 15.51$ per administration in hospital. Additionally, the NHS is required to pay value-added tax (VAT) on all drugs that are administered within a hospital setting (VAT is not incurred for home deliveries). Therefore, these additional costs were also incorporated in the analysis. The VAT rate in the UK in 2020 was $20 \%$, and this was applied to the relevant drug prices for each intervention to estimate the total VAT costs associated with drug administration. As noted previously, PAS discounts are in place for a number of interventions, but as these are confidential, it was necessary to use the list price for all interventions, as reported in the BNF [19].

As with home delivery, the total cost of drug administration for each intervention in the hospital was determined by the dosing frequency for each intervention. All inputs are detailed in Table 1. All costs were based on the 2019/2020 cost year, where possible. An illustration of the model is provided in Fig. 1.

\footnotetext{
1 These costs were obtained following direct communication with a homecare company who provided approximate mean costs for the delivery of biologic interventions for psoriasis.
}

All data in the analysis are based on previously conducted studies, and the analysis does not contain any new studies with human participants or animals performed by any of the authors.

\section{RESULTS}

The analysis considers two methods for drug delivery of biologic interventions for patients with plaque psoriasis: home delivery paid by the NHS and administration in hospitals. The results are presented in Table 2 . The total cost of home delivery over a 2-year time horizon varied substantially based on the choice of intervention. The lowest cost of $£ 693$ occurred with risankizumab, tildrakizumab and ustekinumab. The highest costs of $£ 3445$ occurred with adalimumab, brodalumab, certolizumab and etanercept. Risankizumab, tildrakizumab and ustekinumab are administered once every 12 weeks, which is substantially less frequently than every 1 or 2 weeks, which is the administration frequency associated with adalimumab, brodalumab, certolizumab and etanercept. As such, there is a clear relationship between the dosing schedule and total cost of home delivery.

For the scenario in which the interventions were administered in a hospital setting, the lowest cost of $£ 4224$ was associated with ustekinumab and the highest cost of $£ 7463$ was associated with brodalumab (2-year values). It should be noted that, in this scenario, the largest driver of the overall costs was the drug VAT, and the staff time required for the administration to be completed was secondary.

As the results of the drug administration in hospital method (Table 2) are higher than those of the drug delivery paid by NHS method, with the exception of adalimumab and etanercept, the outputs from this analysis indicate that the NHS could save money by paying for drugs to be delivered to a patient's home, rather than being administered in a hospital setting, due to the associated VAT savings. 
Table 1 Key model inputs

\begin{tabular}{lcl}
\hline Parameter & Value & Source \\
\hline Total administration episodes over a & 2-year period \\
Adalimumab & 52 & Estimated based on dosing schedules described on the British National Formulary \\
Brodalumab & 52 & (includes initial induction period, which may be associated with additional doses) \\
Certolizumab & 52 & {$[19]$} \\
Etanercept & 104 & \\
Guselkumab & 14 & \\
Ixekizumab & 30 & \\
Risankizumab & 10 & \\
Secukinumab & 29 & \\
Tildrakizumab & 10 & \\
Ustekinumab & 10 & \\
Home delivery paid by NHS & \\
Unit cost of drug delivery & $£ 65.00$ & Data provided by Almirall \\
Unit cost of nurse & $£ 140.00$ & \\
Average time for first & 1.00 & Assumption \\
nurse visit & & \\
Average time for & 0.00 & \\
subsequent nurse visit & & \\
Administration in hospital & & \\
Unit cost of nurse & $£ 47.00$ & PSSRU 2019 [20] \\
Time required (hours) & 0.33 & \\
\hline
\end{tabular}

NHS National Health Service, PSSRU Personal Social Services Research Unit

${ }^{a} \mathrm{NB}$ : these costs were obtained following direct communication with a homecare company who provided approximate mean costs for the delivery of biologic interventions for psoriasis

\section{CONCLUSION}

When selecting which biologic intervention to administer to plaque psoriasis patients, the cost of drug delivery is generally overlooked in the total cost. The authors of this article believe this is the first analysis that explores the impact of the cost of drug delivery for biologic interventions paid by the NHS compared with the cost of drug administration in hospitals for plaque psoriasis in the UK.
The results of the analysis indicate that the associated costs with drug delivery are likely to be meaningful and, therefore, should be given greater consideration in the selection process of treatments for plaque psoriasis. This is particularly relevant given the large number of biologic interventions that are available to physicians in the UK for this indication. More specifically, the results show that drug delivery costs are lower for interventions that have a less frequent dosing schedule, which is to be expected. Additionally, with lower-frequency drugs, this 
COST OF HOME DELIVERY

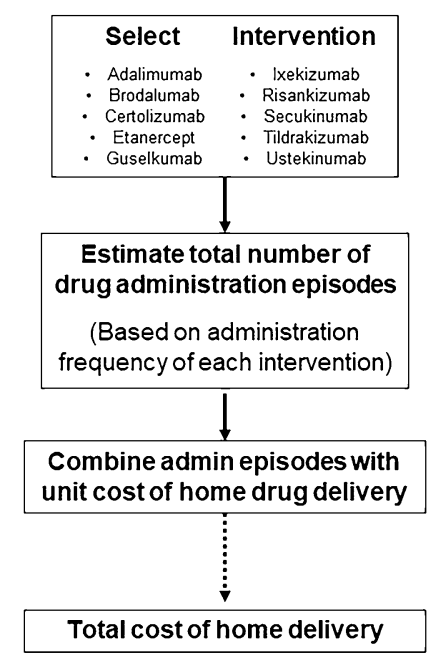

COST OF ADMINISTRATION IN HOSPITAL

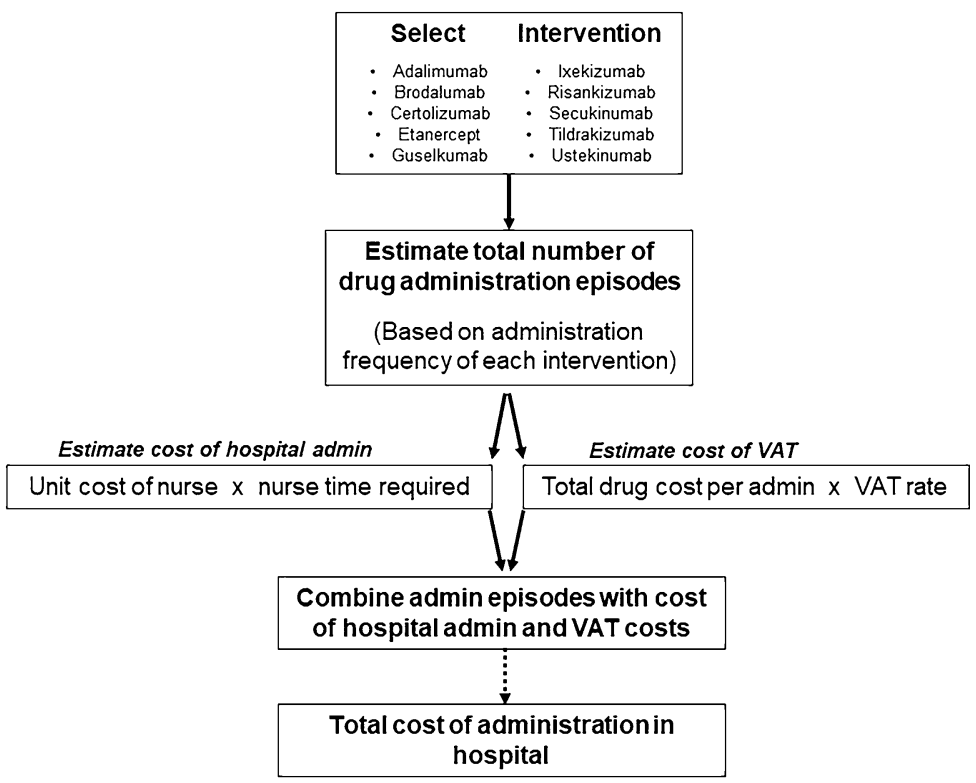

Fig. 1 An overview of the steps undertaken to estimate the total cost of the two options for biologic drug delivery (i.e. delivery at a patient's home or administration within a hospital setting)

analysis suggests that, where biologic agents are administered in the hospital setting for psoriasis, the NHS could save money by switching to a home delivery service as VAT would no longer be incurred on the drug price.

While robust data sources were used for a number of input parameters in the model, the analysis is limited in that there are currently no published data available on the unit costs of home delivery services. The model is thus predicated on assumptions around these variables. Furthermore, as the focus of the analysis was on drug delivery, the potential impact of the cost of the interventions was not considered. This was due to the presence of confidential PAS discounts for a number of interventions included within the analysis. This also had an impact on the VAT costs that were predicted within the model, and as such, the predicted VAT savings by switching to a home delivery service, rather than administration in hospital, will be overestimated to some degree for certain interventions.

While it is important to acknowledge potential limitations, it is valuable to highlight the potential benefits of home drug delivery. Other than the potential cost savings highlighted when interventions with less frequent dosing schedules are chosen, an increase in the use of home delivery systems may also subsequently reduce patient contact with the healthcare system, due to the decreased need for hospital administration of interventions. This reduction in patient contact could lead to other potential benefits that were beyond the scope of the current analysis. In particular, during the COVID-19 pandemic, less patient contact with the healthcare system is likely to reduce the risk of transmission of the infection, which could subsequently lead to many health benefits. There could also be a small productivity gain associated with home delivery if it facilitates less frequent hospital visits.

The delivery of biologic interventions in the treatment of plaque psoriasis is associated with meaningful costs, which are lower for interventions with less frequent dosing schedules, such as risankizumab, tildrakizumab and ustekinumab. Additionally, for interventions with less frequent dosing schedules, the NHS 
Table 2 Base case results - total 2-year costs of drug administration (including induction costs) for drug delivery paid by NHS and drug administration in hospital

\begin{tabular}{|c|c|c|}
\hline Treatment & $\begin{array}{l}\text { Total 2-year cost } \\
\text { of drug delivery } \\
\text { paid by NHS }\end{array}$ & $\begin{array}{l}\text { Total 2-year cost of } \\
\text { drug } \\
\text { administration in } \\
\text { hospital (including } \\
\text { VAT) }\end{array}$ \\
\hline $\begin{array}{l}\text { Adalimumab } \\
\text { (biosimilar) }\end{array}$ & $£ 3455$ & $£ 4293$ \\
\hline Brodalumab & $£ 3455$ & $£ 7463$ \\
\hline Certolizumab & $£ 3455$ & $£ 4739$ \\
\hline $\begin{array}{l}\text { Etanercept } \\
\text { (biosimilar) }\end{array}$ & $£ 6835$ & $£ 4895$ \\
\hline Guselkumab & $£ 1001$ & $£ 6634$ \\
\hline Ixekizumab & $£ 1993$ & $£ 7095$ \\
\hline Risankizumab & $£ 693$ & $£ 6467$ \\
\hline Secukinumab & $£ 1928$ & $£ 6967$ \\
\hline Tildrakizumab & $£ 693$ & $£ 6305$ \\
\hline Ustekinumab & $£ 693$ & $£ 4224$ \\
\hline
\end{tabular}

NHS National Health Service, $V A T$ value-added tax

could generate savings by switching to a home delivery service rather than administering the intervention within a hospital setting.

\section{ACKNOWLEDGEMENTS}

Funding. This study and the Journal's Rapid Service Fee were funded by Almirall.

Disclosures. At the time the analysis was undertaken, William Green and Ayeda Nadeem worked for York Health Economics Consortium (YHEC), a consultancy company, which has been commissioned by Almirall to create the economic model and write the manuscript. Richard Stork and Aina Pi Blanque were employees of Almirall. Richard Stork is now an employee of Chiesi Farmaceutici.
Data Availability. All data generated or analysed during this study are included in this published article.

Author Contributions. W.G. and A.N. led both the model design and construction and also the drafting and editing of the manuscript. R.S. and A.B. were actively involved in all stages of the project, including model development and the drafting of the manuscript.

Compliance with Ethics Guidelines. This article is based on previously conducted studies and does not contain any new studies with human participants or animals performed by any of the authors.

Authorship. All named authors meet the International Committee of Medical Journal Editors (ICMJE) criteria for authorship for this article, take responsibility for the integrity of the work as a whole, and have given their approval for this version to be published.

Open Access. This article is licensed under a Creative Commons Attribution-NonCommercial 4.0 International License, which permits any non-commercial use, sharing, adaptation, distribution and reproduction in any medium or format, as long as you give appropriate credit to the original author(s) and the source, provide a link to the Creative Commons licence, and indicate if changes were made. The images or other third party material in this article are included in the article's Creative Commons licence, unless indicated otherwise in a credit line to the material. If material is not included in the article's Creative Commons licence and your intended use is not permitted by statutory regulation or exceeds the permitted use, you will need to obtain permission directly from the copyright holder. To view a copy of this licence, visit http://creativecommons.org/licenses/bync/4.0/. 


\section{REFERENCES}

1. National Institute for Health and Care Excellence. Psoriasis: assessment and management [CG153]. 2012. Available from: https://www.nice.org.uk/ guidance/cg153. Accessed 15 July 2021.

2. National Institute for Health and Care Excellence. Ixekizumab for treating moderate to severe plaque psoriasis - Final Scope. 2016. Available from: https://www.nice.org.uk/guidance/ta442/history. Accessed 15 July 2021.

3. Springate DA, Parisi R, Kontopantelis E, Reeves D, Griffiths CEM, Ashcroft DM. Incidence, prevalence and mortality of patients with psoriasis: a U.K. population-based cohort study. $\mathrm{Br} \mathrm{J}$ Dermatol. 2017;176(3):650-8.

4. Boehncke WH, Schön MP. Psoriasis. Lancet. 2015;386(9997):983-94.

5. Damiani G, Cazzaniga S, Conic RRZ, Naldi L, Psocare Registry Network. Pruritus characteristics in a large Italian cohort of psoriatic patients. J Eur Acad Dermatol Venereol. 2019;33(7):1316-24.

6. British Association of Dermatologists. Psoriasis-an overview. Available from: https://www.bad.org.uk/ healthcare-professionals/psoriasis. Accessed 15 July 2021.

7. Nast A, Amelunxen L, Matthias A, Boehncke WH, Dressler C, Gaskins M, et al. S3 Guideline for the treatment of psoriasis vulgaris, update-short version part 1-systemic treatment. J German Soc Dermatol. 2018;16(5):645-69.

8. Baliwag J, Barnes DH, Johnson A. Cytokines in psoriasis. Cytokines. 2015;73(2):342-50.

9. East of England NHS Collaborative Procurement Hub. What are homecare medicines services? Available from: https://www.eoecph.nhs.uk/Whatare-Homecare-Medicines-Services.htm. Accessed 15 July 2021.

10. National Institute for Health and Care Excellence. COVID-19 rapid guideline: dermatological conditions treated with drugs affecting the immune response [NG169]. 2020. Available from: https:// www.nice.org.uk/guidance/ng169. Accessed 15 July 2021.
11. National Institute for Health and Care Excellence. Etanercept and efalizumab for the treatment of adults with psoriasis [TA103]. 2006. Available from: https://www.nice.org.uk/guidance/ta103. Accessed 15 July 2021.

12. National Institute for Health and Care Excellence. Adalimumab for the treatment of adults with psoriasis [TA146]. 2008. Available from: https://www. nice.org.uk/guidance/ta146. Accessed 15 July 2021.

13. National Institute for Health and Care Excellence. Ustekinumab for the treatment of adults with moderate to severe psoriasis [TA180]. 2009. Availavle from: https://www.nice.org.uk/guidance/ ta180. Accessed 15 July 2021.

14. National Institute for Health and Care Excellence. Secukinumab for treating moderate to severe plaque psoriasis [TA350]. 2015. Available from: https:// www.nice.org.uk/guidance/ta350. Accessed 15 July 2021.

15. National Institute for Health and Care Excellence. Ixekizumab for treating moderate to severe plaque psoriasis [TA442]. 2017. Available from: https:// www.nice.org.uk/guidance/ta442. Accessed 15 July 2021.

16. National Institute for Health and Care Excellence. Guselkumab for treating moderate to severe plaque psoriasis [TA521]. 2018. Available from: https:// www.nice.org.uk/guidance/ta521. Accessed 15 July 2021.

17. National Institute for Health and Care Excellence. Tildrakizumab for treating moderate to severe plaque psoriasis [TA575]. 2019. Available from: https:// www.nice.org.uk/guidance/ta575. Accessed 15 July 2021.

18. National Institute for Health and Care Excellence. Risankizumab for treating moderate to severe plaque psoriasis [TA596]. 2019. Available from: https:// www.nice.org.uk/guidance/ta596. Accessed 15 July 2021.

19. National Institute for Health and Care Excellence. British National Formulary. Available from: https:// bnf.nice.org.uk/. Accessed 15 July 2021.

20. Curtis L, Burns A. Unit Costs of Health and Social Care 2019, Personal Social Services Research Unit, University of Kent, Canterbury. 